\title{
On Multiple Bases of Eigen and Associated Vectors of Operator Pencils in the Hilbert Spaces
}

\author{
Rakhshanda Dzhabarzadeh \\ Department of functional analysis. Institute of Mathematics and Mechanics of NAS of Azerbaijan, Baku, Azerbaijan
}

Email address:

rakhshanda.dzhabarzade@rambler.ru

\section{To cite this article:}

Rakhshanda Dzhabarzadeh. On Multiple Bases of Eigen and Associated Vectors of Operator Pencils in the Hilbert Spaces. Pure and Applied Mathematics Journal. Special Issue: Spectral Theory of Multiparameter Operator Pencils and Its Applications.

Vol. 4, No. 4-1, 2015, pp. 27-32. doi: 10.11648/j.pamj.s.2015040401.16

\begin{abstract}
It is proved the theorem about of multiple basis of eigen and associated vectors of the operator pencil, non-linear depending on parameter in the Hilbert space. This work is the generalization of existing results on the multiple completeness of the eigen and associated vectors of polynomial pencils, rationally depending on parameters. At the proof the author uses the methods of spectral theory of operators.
\end{abstract}

Keywords: Multiple Basis, Eigen And Associated, Residue, Bounded

\section{Introduction}

Spectral theory of nonselfadjoint operators attracted great attention of mathematicians, physicists, technicians. For a long time the most essential part of this theory remained the study of Hilbert and F.Riss. In theory of nonselfadjoint problems for ordinary differential equations Birkhoff and Tamarkin achieved major successes, based on the study of the analytic properties of the resolvent of the operators. This situation changed only after the publication of the Keldysh's work.(1951). Keldysh M.V. had proved the fundamental result [1] about the multiple completeness of the system of eigen and associated (e.a.) vectors and properties of the eigen values for a wide class of polynomial pencils, like

$$
L(\lambda)=A_{0}+\lambda A_{1} B+\ldots+\lambda^{n-1} A_{n-1} B^{n-1}+\lambda^{n} B^{n}
$$

in the Hilbert space. Keldysh in [1] under the conditions that operators $A_{i}$ are completely continuous; $B$ is positive completely continuous operator of finite order (series of the norms of eigenvalues in some positive degree converges), $\operatorname{Ker} B=\{\vartheta\}$ proved the multiple completeness of the eigen and associated vectors of the operator pencil (1) in the Hilbert space. This result allowed studying of boundary problems for partial differential equations and getting strong results for ordinary differential operator systems which can be applied to the study of equations of mathematical physics and differential equations with partial derivatives.
Later, this result was generalized by many mathematicians in different directions. It should be noted of the works of J..E.Allahverdiev, M.G.Gasymov, A.G.Kostyuchenko, G.Radzievskii and others. The problems of existence of two bases of eigen and associated vectors of the operator $\mu G+\frac{1}{\mu} H$ when $G, H \in \sigma_{\infty} \quad G>0, H \geq 0, \mu-$ complex numbers were considered in the [4],[9].. In these works under some additional conditions was proved the existence of two kind of the bases of the eigen and associated vectors of operator $\mu G+\frac{1}{\mu} H$.

Later, in the work [7] Allakhverdiev proved the existence of two kind of multiple completeness system of eigen and associated vectors of the operator pencil, rationally depending on parameter.

Theorems about multiple expansions of the root subspaces of the operator pencil $L(\lambda)$ were proved in the works of RM Dzhabarzadeh [2], V.N. Vizitey, A.S. Marcus [3] with the proviso that operators $A_{i} B^{-i}$ are bounded if $\frac{\lim }{k \rightarrow \infty} k \mu_{k}^{-p}=0$, and operators $A_{i} B^{-i}$ are completely continuous if $\underline{\lim }_{k \rightarrow \infty} k \mu_{k}^{-p}<\infty$.

Through $\mu_{k}$ are designated the sequence of the characteristic values of the operator $L(\lambda)$, in order of increasing various modules taking into account their 
multiplicities.

In the work [6] it is proved the summation of the series on eigen and associated vectors of the operator pencil (1) by the method of Abel.

The work [5] is devoted also to the questions of multiple summation of series on eigen and associated vectors of operator pencil (1) under the conditions that the resolvent of operator pencil on closed expanding indefinitely contours is uniformly bounded .

Below it is presented a theorem asserting the existing of the multiple basis of eigen and associated vectors of the operator pencil

$$
L(\lambda)=L_{1}(\lambda)+L_{2}(\lambda)
$$

where $\quad L_{1}(\lambda)=A_{0}+\lambda A_{1} B+\ldots+\lambda^{n-1} A_{n-1} B^{n-1}+\lambda^{n} B^{n} \quad$ $L_{2}(\lambda)=C_{0}+\lambda^{-1} C_{1} B+\ldots+\lambda^{-(m-1)} C_{n-1} B^{n-1}+\lambda^{-n} B^{n}$ We show the summation of the root subspaces of the operator pencil $L(\lambda)$, We remind

Definition 1. The sequence of subspaces $\left\{M_{k}\right\}_{k+1}^{\infty}$ is called a basis of subspaces if the any vector $x \in H$ of the space $H$ is

$$
x_{i}=L\left(\lambda_{0}\right) x_{i}+\frac{1}{1 !} \frac{d}{d \lambda} L\left(\lambda_{0}\right) x_{i-1}+\ldots+\frac{1}{i !} \frac{d^{i}}{d \lambda^{i}} L\left(\lambda_{0}\right) x_{0} \quad i=1,2, \ldots k
$$

are satisfied.. Keldysh built the derivative systems according to the rule:

$$
x_{i}^{(j)}=\left[\frac{d^{j}}{d t^{j}} e^{\lambda_{0} t}\left(x_{i}^{(0)}+x_{i-1}^{(0)} \frac{t}{1 !}+x_{i-2}^{(0)} \frac{t^{2}}{2 !}+\ldots+x_{0}^{(0)} \frac{t^{i}}{i !}\right)\right]_{t=0}, j=1,2, \ldots
$$

Definition4. [1] Under canonical system of e.a. vectors for

eigenvalue $\lambda^{0}$ of the operator pencil (1) are understood the system

$$
z^{(k)}, z_{1}^{(k)}, \ldots, z_{p_{k}}^{(k)}(k=1,2, . .)
$$

possessing the following properties: elements $z^{(k)}(k=1,2, .$.$) form basis of a eigen subspace M\left(\lambda^{0}\right)$; there is $z^{(1)}$ eigenvector which multiplicity reaches a possible maxima $p_{1}+1 ; z^{(k)}$ is eigenvector which is not expressing linearly though $z^{(1)}, \ldots, z^{(k-1)}$ which sum of multiplicities reaches a possible maxima $p_{k}+1$.

Let's designate though $M\left(\lambda^{0}\right)$ a subspace spanned by eigen and associated vectors of the system (2), corresponding to an eigenvalue $\lambda^{0}$.

Linearly-independent elements form a chain of a set (5) of eigen and associated (e.a) vectors of (1). The multiplicity of eigenvalue $\lambda^{0}$ designates the greatest number of associated vectors to $z_{0, \ldots, 0}$ a plus 1 .

The sum $p_{1}+p_{2}+\ldots+p_{s}+s$ is a multiplicity of an eigen value $\lambda^{0}$.

Definition5. Under $n$-multiple completeness of eigen and associated vectors of the operator pencil $L(\lambda)$ in space $H$ it is understood the possibility of approaching any $n$ element $f_{0}, f_{1}, \ldots, f_{n-1}$ of the space $H$ by linear combinations of elements $\left\{x_{i}^{(j)}\right\}_{i=1}^{\infty}, j=0,1,2, \ldots, n-1$, respectively, with accuracy given in advance, with the same coefficients for all elements $f_{0}, f_{1}, \ldots, f_{n-1}$.

Remark1.[1].If at least for one point $\lambda=\lambda_{0}$ of the operator $L(\lambda)=I-A_{0}-\lambda A_{1} \ldots-\lambda^{n} A_{n} ;\left(A_{j} \in \sigma_{\infty}\right)$ invertible, then the set of eigenvalues of the pencil $L(\lambda)$ consists of isolated points of finite algebraic multiplicity ([1] and [4)].

\section{The Spectral Theorem on Multiple Basis of Eigen and Associated Vectors of the Operator Pencil}

The study of the spectral properties of the equation $L(\lambda) x=x$ in a Hilbert space $H$ is reduced to the study of the spectral properties of the equations $\bar{A}\left(\lambda^{-1}\right) \bar{x}+\lambda \bar{B} \bar{x}=\bar{x}$ and $\bar{C}(\lambda) \bar{x}+\lambda^{-1} \bar{B} \bar{x}=\bar{x}$ in the direct sum $\bar{H}$ of $n$ copies of the Hilbert space $H$.

The equation

$$
L(\lambda) x=x
$$


can be represented as a system of equations:

$$
\begin{aligned}
& x_{0}=\left(L_{2}(\lambda)+A_{0}\right) x_{0}+A_{1} x_{1}+\ldots+A_{n-1} x_{n-1}+\lambda B x_{n-1} \\
& x_{1}=\lambda B x_{0} \\
& x_{2}=\lambda B x_{1} \\
& \cdots \cdots \cdots \\
& x_{n-1}=\lambda B x_{n-2}
\end{aligned}
$$

and the equation $\bar{A}\left(\lambda^{-1}\right) \bar{x}+\lambda \bar{B} \bar{x}=\bar{x}$, where

$$
\begin{gathered}
\overline{A\left(\lambda^{-1}\right)}=\left(\begin{array}{ccccc}
L_{2}(\lambda)+A_{0} & A_{1} & \ldots & A_{n-1} \\
0 & & 0 & \ldots & 0 \\
. & & . & \ldots & . \\
0 & & 0 & \ldots & 0
\end{array}\right) \\
\bar{B}=\left(\begin{array}{ccccc}
0 & 0 & 0 & \ldots & B \\
B & 0 & 0 & \ldots & 0 \\
0 & B & 0 & \ldots & 0 \\
. & . & . & \ldots & . \\
0 & 0 & 0 & \ldots B & 0
\end{array}\right), \\
\bar{E}=\left(\begin{array}{cccc}
E & 0 & \ldots & 0 \\
0 & E & \ldots & 0 \\
. & . & \ldots & . \\
0 & 0 & \ldots & E
\end{array}\right) .
\end{gathered}
$$

$L(\lambda)$ can be represent as a system of equations

$$
\begin{aligned}
& x_{0}=\left(L_{1}(\lambda)+C_{0}\right) x_{0}+C_{1} x_{1}+\ldots+C_{n-1} x_{n-1}+\lambda^{-1} B x_{n-1} \\
& x_{1}=\lambda^{-1} B x_{0} \\
& x_{2}=\lambda^{-1} B x_{1} \\
& \cdots \cdots \cdots \\
& x_{n-1}=\lambda^{-1} B x_{n-2}
\end{aligned}
$$

And equation $\bar{C}(\lambda) \bar{x}+\lambda^{-1} \bar{B} \bar{x}=\bar{x}$

$$
\left(E-\bar{A}\left(\lambda^{-1}\right)-\lambda \bar{B}\right)^{-1}=(\bar{E}-\lambda \bar{B})^{-1}\left(\bar{E}-\bar{A}\left(\lambda^{-1}\right)(\bar{E}-\lambda \bar{B})^{-1}\right)^{-1}
$$

The characteristic numbers of the operator $\bar{B}$ lie on the rays with the arguments $\frac{2 \pi k}{n}, k=1,2, \ldots, n$.The equalities $\left\|(\bar{E}-\lambda \bar{B})^{-1}\right\|=\max \left|\frac{1}{1-\mu_{k} e^{i \frac{2 \pi k}{n}} \lambda}\right|==\max \left|\frac{1}{1-\mu_{k}\left(e^{i \frac{2 \pi k}{n}} \lambda\right)}\right|$. $e^{i \frac{2 \pi s}{n}} \lambda \in \Gamma_{k}$, if $\lambda \in \Gamma_{k}$, So $\left\|(E-\lambda B)^{-1}\right\|<C$ for all $\lambda \in \Gamma_{k}$ then on the contours $\Gamma_{k}$ norms of the operators $\left\|(\bar{E}-\lambda \bar{B})^{-1}\right\|<C$ satisfy the conditions (6) for all $\lambda \in \Gamma_{k}$. Further, $\left\|\bar{A}\left(\lambda^{-1}\right)(\bar{E}-\lambda \bar{B})^{-1}\right\| \rightarrow 0$ when $k \rightarrow \infty$., (see[4]). where $\bar{C}=\left(\begin{array}{cccc}L_{1}(\lambda)+C_{0} & C_{1} & \ldots & C_{n-1} \\ 0 & 0 & \ldots & 0 \\ . & . & \ldots & . \\ 0 & 0 & \ldots & 0\end{array}\right)$

Operators $\bar{A}, \bar{B}, \bar{C}, \bar{E}$ act in direct sum of $n$ copies of the Hilbert space $H$.

Consider the operator $\bar{A}\left(\lambda^{-1}\right) \bar{x}+\lambda \bar{B} \bar{x}=\bar{x}$

Operator $\bar{A}$ is the completely continuous in the space $H^{n}$ (direct sum of $n$ copies of the space) so the operators $A_{i}, \quad(i=0,1, \ldots, n-1)$ are completely continuous in the space $H$.

So the operator $B$ is a selfadjoint, completely continuous, then the characteristic values of $\bar{B}$ lie on the n rays emanated from origin, norms of characteristic values of operators $\bar{B}$ and $B$ coincide. $\bar{B}$ is a normal completely continuous operator.

Theorem. Suppose that the following conditions are satisfied:

a) operators $A_{i}(0=1,2, \ldots, n-1), C_{i}(i=0,1,2, \ldots, n-1)$ and $B, D$ are complete continuous;

b) there is the sequence of closed concentric circumferences with radii $\rho\left(\Gamma_{k}\right)$ tending to infinity $\rho\left(\Gamma_{k}\right) \rightarrow \infty$, in which for all $\lambda \in \Gamma_{k}$ we have

$$
\left\|(E-\lambda B)^{-1}\right\|<C
$$

Then the eigen values of the operator $L(\lambda)$ can be grouped so that the subspaces spanned by their eigen and associated vectors corresponding to the eigen values form $n$-multiple basis with brackets in the space $H$. All eigen values are divided on two sequences; one of them has the limit in infinity, other has the limit point in zero.

Proof of Theorem.

We evaluate the resolvent-operator $\left(E-\bar{A}\left(\lambda^{-1}\right)-\lambda \bar{B}\right)^{-1}$ for the values $\lambda \in \Gamma_{k}$. Let $\lambda \in \Gamma_{k}$ then.
So $\left\|\bar{A}(\bar{E}-\lambda \bar{B})^{-1}\right\|<q<1$, then starting with some $k_{1}$. $\left(\bar{E}-\bar{A}\left(\bar{E}-\lambda \bar{B}^{-1}\right)^{-1}=1+\bar{A}(\bar{E}-\lambda \bar{B})^{-1}+\left(\bar{A}(\bar{E}-\lambda \bar{B})^{-1}\right\}^{2}+\ldots\right.$

$$
\begin{gathered}
\left\|\left(\bar{E}-\overline{A\left(\lambda^{-1}\right)}(\bar{E}-\lambda \bar{B})^{-1}\right)^{-1}\right\|<\frac{1}{1-q} \\
\left\|\left(\bar{E}-\bar{A}\left(\lambda^{-1}\right)-\lambda \bar{B}\right)^{-1}\right\| \leq \frac{1}{1-q}\left\|(\bar{E}-\lambda \bar{B})^{-1}\right\| \leq C_{1}
\end{gathered}
$$

By analogy we evaluate the resolvent of the operator $\bar{E}-\bar{C}(\lambda)-\lambda^{-1} \bar{B}$ when $\frac{1}{\lambda} \in \Gamma_{k}$, Denote $\frac{1}{\lambda}=\mu$ then we 
obtain the equation $\left(\bar{E}-\bar{C}\left(\mu^{-1}\right)-\mu \bar{B}\right) \bar{y}=0$ where $\mu \in \Gamma_{k}$, Then starting with some $k_{0}$ for $\mu \in \tilde{\Gamma}_{k},\left(k>k_{2}\right)$

$$
\left\|\left(\bar{E}-\overline{C\left(\mu^{-1}\right)}(\bar{E}-\overline{\mu B})^{-1}\right)^{-1}\right\|<\frac{1}{1-q_{1}}
$$

and

$$
\left.\|\left(\bar{E}-\overline{C\left(\mu^{-1}\right)}-\overline{\mu B}\right)^{-1}\right)^{-1} \|<C_{1}
$$

Denote though $G_{k}$ the domain of the complex plain bounded by contour $\Gamma_{k}$ and $\Gamma_{k-1}$ and though $\gamma_{k}$ the contour, bounded the domain $G_{k}$.

We introduce the operator by the formula

$$
\bar{P}_{k} \bar{f}=\frac{1}{2 \pi i} \int_{\gamma_{k}}\left(\bar{E}-\bar{A}\left(\lambda^{-1}\right)-\lambda \bar{B}\right)^{-1} / \lambda^{2} \bar{f} d \lambda
$$

$\gamma_{k}$ is the border of the of the domain $G_{k}$..It is known that the meaning of this integral is equal to the sum of residues respectively of all poles of integrand, lying within the domain $G_{k}$.

Within the domain $G_{k}$, bounded by the contours $\Gamma_{k}$ and $\Gamma_{k+1}$, the integrand has the poles in characteristic values of the operator $\overline{(E}-\lambda\left(\bar{E}-\bar{A}\left(\lambda^{-1}\right)\right)^{-1} \bar{B}^{-1}$. Let $\lambda=\lambda_{1}$ be a pole of the integrand in (11). The general part of the resolution of the operator $\left.\overline{(E}-\lambda\left(\bar{E}-\bar{A}\left(\lambda^{-1}\right)\right)^{-1} \bar{B}\right)$ at the neighborhood of its isolated characteristic value $\lambda=\lambda_{1}$ has the form $\sum_{k} \sum_{0 \leq s \leq m_{k}} \frac{\sum_{0 \leq i \leq s} \bar{x}_{i}^{(k)} z_{s-i}^{(k)}}{\left(\lambda-\lambda_{i}\right)^{m_{k}-s+1}}$

In the representation

$$
\sum_{k} \sum_{0 \leq s \leq m_{k}} \frac{\sum_{0 \leq i \leq s} x_{i}^{-(k)} z_{s-i}^{-(k)}}{\left(\lambda-\lambda_{i}\right)^{m_{k}-s+1}}
$$

$\left\{x_{i}^{k}\right\}_{i=1}^{\infty}$ is the canonical system of eigen and associated vectors of the operator pencil $\left(\bar{E}-\bar{A}\left(\lambda^{-1}\right)-\lambda \bar{B}\right)^{-1}, \bar{z}_{s}^{(k)}$ is a canonical system of eigen and associated system of adjoint to $\left(\bar{E}-\bar{A}\left(\lambda^{-1}\right)-\lambda \bar{B}\right)^{-1}$ operator $\left[\left(\bar{E}-\bar{A}\left(\lambda^{-1}\right)-\lambda \bar{B}\right)^{-1}\right]^{*}$, product $\bar{x}_{i}^{(k)} z_{s-i}^{(k)}$ is the operator which on the element $\bar{f} \in \bar{H}$ is defined by the rule $\bar{S} \bar{f}=(\bar{f}, \bar{z}) \bar{x}$. In the neighborhood of the isolated eigen value $\lambda_{i}$ of the operator $\overline{(E}-\lambda\left(\bar{E}-\bar{A}\left(\lambda^{-1}\right)\right)^{-1} \bar{B}^{-1}$ the general part of resolution of integrand be $\sum_{k} \sum_{0 \leq s \leq m_{k}} \frac{\sum_{0 \leq i \leq s} \bar{x}_{i}^{-(k)} z_{s-i}^{(k)}}{\lambda_{1}^{2}\left(\lambda-\lambda_{i}\right)^{m_{k}-s+1}}$ and residue of integrand in its poles $\lambda_{i}$ is $\frac{\bar{x}_{0}^{(k)} z_{m_{k}}+\ldots+\bar{x}_{m_{k}}^{(k)} z_{0}(k)}{\lambda_{1}^{2}}$

Evaluate the integral

$$
\bar{P}_{k} \bar{f}=\frac{1}{2 \pi i} \int_{\gamma_{k}}\left(\bar{E}-\bar{A}\left(\lambda^{-1}\right)-\lambda \bar{B}\right)^{-1} / \lambda^{2} \bar{f} d \lambda
$$

On the contours $\Gamma_{k}$ we have

$\left\|\bar{P}_{k} \bar{f}\right\| \leq\left|\frac{1}{2 \pi}\right| \int_{\gamma_{k}}\left\|\left(\bar{E}-\bar{A}\left(\lambda^{-1}\right)-\lambda \bar{B}\right)^{-1}\right\| /\left|\lambda^{2}\left\|\left.|\bar{f} \|| d \lambda\left|\leq C_{1}\right| \lambda\right|^{-1}\right.\right.$

In fact it is possible to choose the contours $\Gamma_{k}$ such manner that

$$
\sum_{k=k_{0}}^{\infty}\left\|P_{k+1}-P_{k}\right\|<\infty
$$

Adopted $\mu=\frac{1}{\lambda}, \mu \in \Gamma_{k}$ we consider the integral

$$
\overline{\tilde{P}}_{k} \bar{f}=\frac{1}{2 \pi i} \int_{\gamma_{k}}\left(\bar{E}-\bar{C}\left(\mu^{-1}\right)-\mu \bar{B}\right)^{-1} / \mu^{2} \bar{f} d \mu
$$

The contours $\gamma_{k}$ are the border of $G_{k}$.It is not difficult to see all poles of integrand in (15), starting with some $k_{s}$ lie in the domain $G_{k}$ so they are the characteristic values of the operator $L(\lambda)$. Then the poles of integrand of (13) and (15) in the $G_{k}$, starting with some $k_{0}$, coincide. Moreover, the same residues of the corresponding same poles also coincide. We can choose the sequence of contours $\gamma_{k}$ for which $\sum_{k=k_{0}}^{\infty}\left\|\tilde{P}_{k+1}-\tilde{P}_{k}\right\|<\infty$, thus of the subspaces spanned by eigen and associates vectors, corresponding to the eigen of values from $G_{k}, G_{k+1}, \ldots$ form the basis with brackets in the space spanned by the eigen and associated vectors starting with sufficiently great index $k$.

Introduce the contours $\tilde{\Gamma}_{k}$ and $\tilde{\Gamma}_{k-1}$ where $\tilde{\Gamma}_{k}$ is the set of numbers $\lambda=\frac{1}{\mu}$, where $\mu \in \Gamma_{k}$ and $\tilde{\Gamma}_{k-1}$ is the set of numbers $\mu=\frac{1}{\lambda}, \lambda \in \tilde{\Gamma}_{k-1}$ Denote though $\tilde{G}_{k}$ the domain bounded by the contours $\tilde{\Gamma}_{k}$ and $\tilde{\Gamma}_{k-1}$ and though the $\tilde{\mu}_{k}$ the contour bounded the domain $\tilde{G}_{k}$.

The sum of residues of all poles of integrand in (15) coincides with the subspace spanned by the eigen and associated vectors corresponding to all eigen values lying in the domain $\tilde{G}_{k}$.

So we have two sequences of eigen values of the operator $L(\lambda)$, one sequence of eigenvalues of $L(\lambda)$ tends to infinity, and another tends to zero. Denote though $G$ the area of the 
complex plain, bounded by the contours $\Gamma_{k_{0}}$ and $\tilde{\Gamma}_{k_{0}}$. It is known that in $G$ there are the finite number of eigen values of each operators $\bar{E}-\bar{A}\left(\lambda^{-1}\right)-\lambda \bar{B}$ and $\bar{E}-\bar{C}(\lambda)-\lambda^{-1} \bar{B}$ and also $L(\lambda)$.

The point zero does not enter the domain $G$. The sum of all residues of the all poles integrand in the domain $G$ contains the finite number of terms .

Thus we have $\sum_{k=k_{0}}^{\infty}\left\|P_{k+1}-P_{k}\right\|<\infty$ and $\sum_{k=k_{0}}^{\infty}\left\|\tilde{P}_{k+1}-\tilde{P}_{k}\right\|<\infty$,

Operator $\tilde{P}_{k+1}-\tilde{P}_{k}$ is the project of the $\bar{H}$ on the subspace spanned by the eigen and associated vectors of the operator $\bar{E}_{1}-\bar{C}(\lambda)-\lambda^{-1} \bar{B}$, lying in the $\tilde{G}_{k}$. We add to the considering set of subspaces the residue of the $\lambda=0$ and the subspace spanned by the eigen and associated vectors, corresponding to all characteristic values from $G$. Last means the existence of n multiple basis in the space $\bar{H}$ For the definition the residue of integrand in zero in the small neighborhood $G_{0}$ with the border $\delta$ of the point $\lambda=0$ consider the integral

$$
\overline{\tilde{P}}_{k} \bar{f}=\frac{1}{2 \pi i} \int_{\delta}\left(\bar{E}-\bar{C}(\lambda)-\lambda^{-1} \bar{B}\right)^{-1} / \lambda^{2} \bar{f} d \lambda
$$

when $\lambda^{-1} \in \Gamma_{k}$

We have $\bar{E}-\bar{C}(\lambda)-\lambda^{-1} \bar{B}=\lambda^{-1}\left(\lambda \overline{E_{1}}-\lambda \bar{C}(\lambda)-\bar{B}\right)$ and $\left(\bar{E}-\bar{C}(\lambda)-\lambda^{-1} \bar{B}\right)^{-1}=\lambda\left(\lambda \overline{E_{1}}-\lambda \bar{C}(\lambda)-\bar{B}^{-1}\right.$. Substituted the last expression into (16) we obtain

$$
\begin{gathered}
\overline{\tilde{P}}_{0} \bar{f}=\frac{1}{2 \pi i} \int_{\delta}\left(\bar{E}-\bar{C}(\lambda)-\frac{1}{\lambda} \bar{B}\right)^{-1} / \lambda^{2} \bar{f} d \lambda=\quad \begin{array}{r}
\text { system }\left\{\bar{x}_{i}=\left(x_{0, i}, x_{1, i}, \ldots, x_{n-1, i}\right)\right\}_{1}^{\infty} \text { of } \\
\text { defined with the help of the formulas }
\end{array} \\
x_{k, i}=B^{k}\left(\lambda^{k} x_{0, i}+k \lambda^{k-1} x_{0, i-1}+\frac{k(k-1)}{2 !} \lambda^{k-2} x_{0, i-2}+\ldots+\frac{k(k-1) \ldots 1}{k !} x_{0,0}\right)
\end{gathered}
$$

-the assertion about possibility of expansion of elements from range of operator $\bar{B}$ on eigen and associated vectors of the operator $\bar{L}(\lambda)$. For the completing of the proof of this theorem we need in the statement of connections between the eigen and associated vectors of operators $\overline{(E}-\bar{A}\left(\lambda^{-1}\right)-\lambda \bar{B}^{-1}$ and $L(\lambda)$.

It is not difficult to state that the first components of eigen and associated vectors of the operator $\bar{E}-\overline{A\left(\lambda^{-1}\right)}-\lambda \bar{B}$ coincide with the eigen and associated vectors of the equation (1), correspondingly.

If $\left\{\bar{x}_{i}=\left(x_{0, i}, x_{1, i}, \ldots, x_{n-1, i}\right)\right\}_{1}^{\infty}$ is the system of eigen and associated vectors of (2) then $\left\{x_{0, i}\right\}_{1}^{\infty}$ is the system of eigen and associated vectors of the operator $L(\lambda)$. Components $x_{k, i}(k=1,2, \ldots, n-1)$ of eigen and associated vectors of the system $\left\{\bar{x}_{i}=\left(x_{0, i}, x_{1, i}, \ldots, x_{n-1, i}\right)\right\}_{1}^{\infty}$ of the operator $\bar{L}(\lambda)$ are where $\left\{x_{0, i}\right\}$ is the chain of eigen and associated vectors of the operator $L(\lambda)$. So the closure of the range of the operator $\bar{B}$ coincides with the whole space $\bar{H}$, then the completeness of the eigen and associated vectors of the operator $\bar{L}(\lambda)$ in the space $\bar{H}$ takes place. The last means the $n$ - multiple completeness of eigen and associated vectors of the pencil $L(\lambda)$ in the Hilbert space $H$. Moreover, element from the range of the operator $\bar{B}$ is expanded on the system of the eigen and associated vectors of the operator $\bar{L}(\lambda)$ in the space $\bar{H}$.So we proved the possibility of $n$ - multiple expansions on the system of eigen and associated vectors of the operator $L(\lambda)$ with brackets for any $n$ - elements $f_{0}, f_{1}, \ldots, f_{n-1} ; f_{i} \in R(B)$ of the space $H$.

The Theorem is proved.

\section{Conclusion}

It is proved the convergence of $\mathrm{n}$ series on eigen and associated vectors of operator pencil rationally depending on parameters in the Hilbert space.

\section{References}

[1] Keldysh M.V. About completeness of eigen functions of some class linear non-selfadjoint operators. Journal:Success of Mathematical Science, 1971, т.27, issue.4, pp.15-47

[2] Dzhabarzadeh R.M. On expansions series on eigen and associated vectors of operator pencils, Journal: Scientific notes of Azerb.State University,1964, №3,pp.75-81.

[3] Vizitei V.N., Markus A.S.. On convergence of multiple expansions on the system of eigen and associated vectors of polynomial pencils Mathematical collection,1965,т.66, №2,pp..287-320 
[4] Gokhberg I. Ts., Kreyn M.Q. Introduction to the theory of linear non-selfadjoint operators in the Hilbert space.Moscow, 1964, pp 1-433

[5] Allakhverdiev J.E., Dzhabarzadeh R. M. // Spectral theory of operator pencil in the Hilbert space.ДAN of Azerbaijan - 2011, т.LXVII, № 4.- pp.3-10

[6] Allakhverdiev J.E. Dzhabarzadeh R.M. On summation of multiple series on eigen and associated vectors operator pensilw by Abel?s method. ДAN Az. SSR, 1979,т.35, № 7, p 19-23..
[7] Allakhverdiev J E. The evolution of resolvents and the theorems on completeness of oerators, depending on spectral parameters. Transaction of AN of Azerb. SSR, seria of physics -tekhnics and mathematical sciences, 1074,6,pp.3-36

[8] Lidskii. About summation of the series on the general vectors of the non-selfadjoint operators. Proceeding of Moscow Scientific Society, t.11,1962

[9] Askerov N.Q.,Kreyn S.Q., Laptev Q.I. On some class of nonselfadjoint boundary problems. DAN, 155, 3 (1964),499-502 